\title{
bFGF expression is differentially regulated by cocaine seeking versus extinction in learning-related brain regions
}

\author{
Elizabeth M. Doncheck, ${ }^{1,3}$ Madalyn Hafenbreidel, ${ }^{1,3}$ Sarah A. Ruder, ${ }^{1}$ \\ Michael K. Fitzgerald, ${ }^{1}$ Lilith Torres, ${ }^{2}$ and Devin Mueller ${ }^{1,2}$ \\ ${ }^{1}$ Department of Psychology, University of Wisconsin-Milwaukee, Milwaukee, Wisconsin 53201-0413, USA; ${ }^{2}$ Department of Basic \\ Sciences, Ponce Health Sciences University-School of Medicine/Ponce Research Institute, Ponce, Puerto Rico, 00732, USA
}

\begin{abstract}
In cocaine use disorder, relapse can be elicited by drug-associated cues despite long periods of abstinence. The persistence of drug-associated cues in eliciting drug seeking suggests enduring changes in structural and functional plasticity, which may be mediated by basic fibroblast growth factor (bFGF, FGF2). Stimulant drug use increases bFGF expression in reward- and learning-related brain regions, such as the infralimbic medial-prefrontal cortex (IL-mPFC), and we previously found that this increase was reversed by extinction. However, whether bFGF expression is similarly modified in other brain regions is unknown. Therefore, we used the conditioned place preference (CPP) paradigm to assess bFGF expression following cocaine-associated CPP or extinction of that CPP within the mPFC, nucleus accumbens (NAc), hippocampus (Hipp), and basolateral amygdala (BLA). bFGF expression was increased in IL-mPFC and NAc-Core and -Shell following a cocaine-associated CPP, an effect reversed by extinction. Conversely, bFGF expression was increased in BLA following extinction, but no significant changes were observed in PL-mPFC or either dorsal or ventral Hipp. These results demonstrate differential regulation of bFGF following cocaine-associated CPP or extinction of that CPP in discrete brain regions. Changes in bFGF expression may regulate long-lasting drug-induced plasticity that underlies persistent drug-associated memories, and therefore present potential prophylactic targets.
\end{abstract}

There are currently no FDA-approved treatments for cocaine use disorder, and individuals who abuse cocaine remain vulnerable to relapse despite treatment or long periods of abstinence (McLellan et al. 2000). This long-lasting vulnerability is maintained by exposure to cues associated with the drug, which can promote craving (Ehrman et al. 1992) and lead to relapse (Kosten et al. 2006). Although the longevity of drug-associated memories suggests persistent plastic changes, characterizations of such changes are lacking. Drug use can induce increases in neurite outgrowth and spine density in brain regions such as the prefrontal cortex (PFC) and nucleus accumbens (NAc; Robinson and Kolb 1997, 1999). However, whether such changes directly subserve persistent drug-associated memories, or result from drug exposure generally, is currently unknown.

One probable regulator of drug-induced neural plasticity is basic fibroblast growth factor (bFGF, FGF2). Repeated stimulant administration increases bFGF expression in the infralimbic medial PFC (IL-mPFC; Hafenbreidel et al. 2015), striatum, hippocampus (Maggio et al. 1998; Roceri et al. 2001; Fumagalli et al. 2006), and ventral tegmental area (VTA; Flores et al. 1998). As a growth factor, the drug-induced increase in bFGF expression may promote plastic changes. For example, in VTA, bFGF is necessary for amphetamine-induced neurite outgrowth (Mueller et al. 2006), which is a persistent form of plasticity seen in other reward-related brain regions (Robinson and Kolb 1997, 1999). Besides drug-induced plasticity, bFGF is required for learning and memory. Increased bFGF mRNA in the dentate gyrus corresponds with learning (Gómez-Pinilla et al. 1998), and bFGF administration improves

\footnotetext{
${ }^{3}$ These authors contributed equally to this work. Corresponding author: devin.mueller@gmail.com

Article is online at http://www.learnmem.org/cgi/doi/10.1101//m.047530.118. Freely available online through the Learning \& Memory Open Access option.
}

learning and alleviates memory-related cognitive impairments (Srivastava et al. 2008). Moreover, bFGF facilitates both the acquisition (Graham and Richardson 2009b) and extinction (Graham and Richardson 2009a) of contextual fear conditioning, and reduces reinstatement when administered immediately following extinction either systemically (Graham and Richardson 2010) or directly within the basolateral amygdala (BLA; Graham and Richardson 2011b). Thus, bFGF has an important role not only in drug-induced structural changes, but in learning and memory as well.

Although bFGF is important for learning and memory, stimulant drug use results in an overexpression of bFGF (Flores et al. 1998; Fumagalli et al. 2006; Hafenbreidel et al. 2015), which can have behavioral consequences. bFGF is required for amphetamine-induced behavioral sensitization (Flores et al. 2000) and neutralizing bFGF in IL-mPFC facilitates extinction of cocaine seeking following self-administration (Hafenbreidel et al. 2015). Interestingly, extinction itself can reverse drug-induced increases in bFGF expression in IL-mPFC (Hafenbreidel et al. 2015). However, whether other reward- and learning-related brain regions demonstrate similar changes in bFGF expression or whether extinction can ameliorate these changes is unknown. Therefore, we used the cocaine conditioned place preference (CPP) paradigm, wherein rats were conditioned to associate a distinct context with the rewarding effects of the drug, to examine changes in bFGF expression within reward- and learning-related brain circuitry following both conditioning and extinction.

(C) 2018 Doncheck et al. This article, published in Learning \& Memory, is available under a Creative Commons License (Attribution-NonCommercial 4.0 International), as described at http://creativecommons.org/licenses/by-nc/4.0/. 


\section{Results}

\section{Extinction reverses cocaine-induced} increases in bFGF expression in IL-mPFC and NAC

The effects of extinction of cocaine CPP on bFGF expression were examined by measuring bFGF immunoreactivity in reward- and learning-related brain regions. Following an initial pretest, rats were matched into three groups with no overall chamber bias. Next, rats were conditioned to associate one chamber, but not another, with cocaine $(10 \mathrm{mg} / \mathrm{kg}$, i.p.), and a control group was conditioned to associate both chambers with saline. Following conditioning, some rats (SalExt and Coc-Ext) underwent two 30-min extinction sessions, whereas others (CocNoExt) remained in their home cages (Fig. 1A). To determine if rats demonstrated a preference for the cocaine-paired chamber following conditioning, extinction day 1 was analyzed with a one-way ANOVA. As expected, following conditioning, Sal-Ext rats did not express a CPP during the first extinction session (Fig. $\left.1 \mathrm{~B} ; F_{(2,9)}=1.38, P=\mathrm{ns}\right)$. Coc-NoExt rats did not undergo extinction testing (Fig. 1C; denoted in the figure as blank CPP extinction sessions). In contrast, Coc-Ext rats expressed a CPP for the previously cocaine-paired chamber during the first extinction session (Fig. 1D; $F_{(2,15)}=$ $9.92, P=0.002)$. Post hoc tests confirmed that rats spent more time in the previously cocaine-paired chamber than salinepaired chamber $(P=0.007)$, indicating that rats expressed a cocaine CPP. Next, to determine if rats demonstrated extinction, extinction day 2 was analyzed with a one-way ANOVA. During the second extinction session, Sal-Ext rats again did not express a significant $\operatorname{CPP}\left(F_{(2,9)}=4.11, P=\right.$ ns). Similarly, Coc-Ext rats did not express a CPP during the second extinction session $\left(F_{(2,15)}=0.40, P=\mathrm{ns}\right)$, demonstrating that extinction had occurred.

The next day, all rats were tested for extinction during a 30-min session (Fig. 1E). Sal-Ext rats again did not express a significant CPP, as a one-way ANOVA revealed no significant effect of chamber $\left(F_{(2,9)}=1.20, P=\mathrm{ns}\right)$. Similarly, Coc-Ext rats did not express a cocaine CPP, demonstrating extinction recall, as a one-way ANOVA revealed no significant effect of chamber $\left(F_{(2,15)}=2.11\right.$, $P=\mathrm{ns}$ ). However, Coc-NoExt rats did express a cocaine CPP, as a one-way ANOVA revealed a significant effect of chamber $\left(F_{(2,15)}=9.30, \quad P=0.002\right)$. Post hoc tests confirmed that CocNoExt rats spent more time in the previously cocaine-paired chamber than in the saline-paired chamber during the extinction test $(P=0.001)$, indicating that rats expressed a cocaine CPP. Importantly, Coc-NoExt rats did not demonstrate within

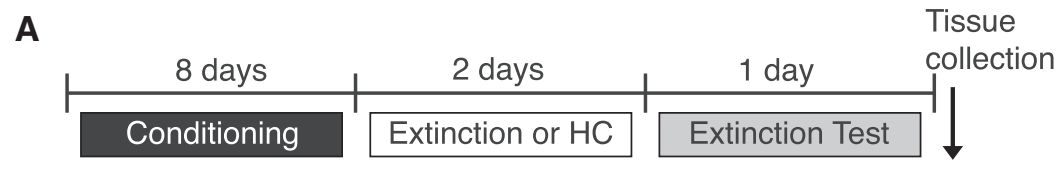

B

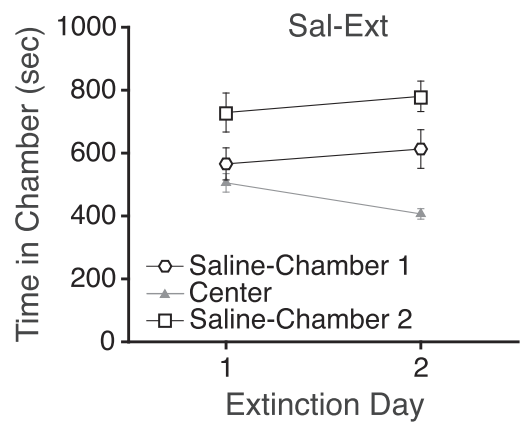

D
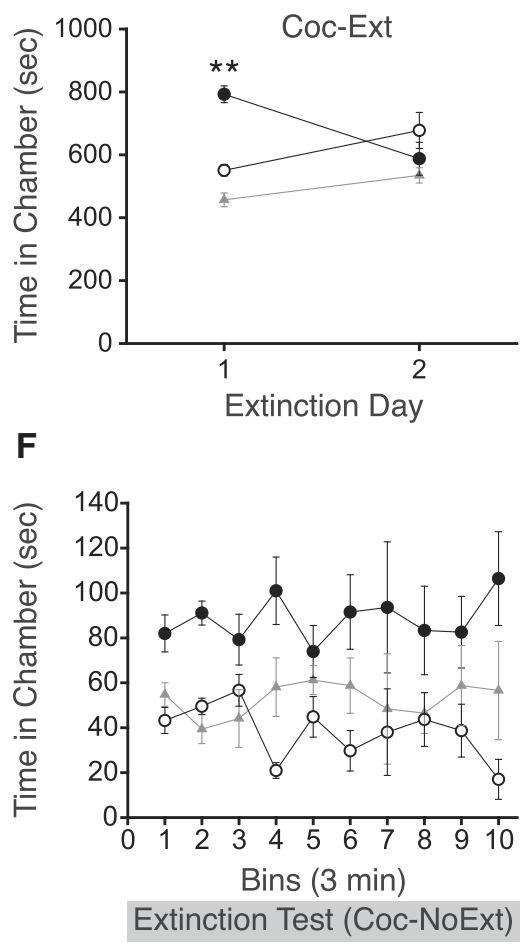

C

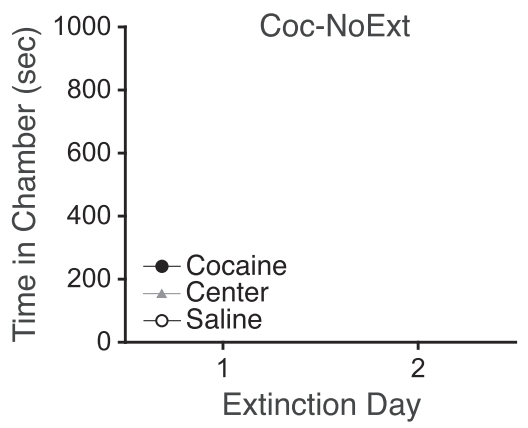

E

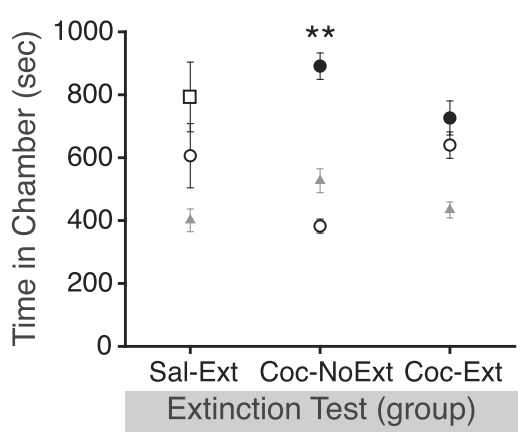

Figure 1. Extinction and extinction test prior to quantifying bFGF-immunoreactivity. $(A)$ Schematic showing design of CPP conditioning and testing. (B) Sal-Ext rats had no significant preference for either saline-paired chambers during extinction day 1 and 2. (C) Coc-NoExt rats remained in their home cages while the other two groups underwent extinction, and therefore did not undergo extinction or receive exposure of the conditioning contexts. No behavioral data was collected for these rats during extinction day 1 and 2 (denoted as a blank space). (D) Coc-Ext rats had a significant preference for the previously cocaine-paired chamber during extinction day 1 , but not extinction day 2. (E) Coc-NoExt rats had a significant preference for the previously cocaine-paired chamber during the extinction test, whereas Sal-Ext and Coc-Ext rats did not. $(F)$ Coc-NoExt rats do not demonstrate within session extinction during the extinction test. Error bars are \pm SEM. $\left({ }^{* *}\right) P<0.01$. HC, Home Cage. session extinction when the 30-min extinction test was analyzed in 3-min bins (Fig. 1F). A two-way repeated measures ANOVA revealed no significant effect of bin $\left(F_{(9,150)}=0.01, P=\mathrm{ns}\right)$, or chamber by bin interaction $\left(F_{(18,150)}=0.79, P=n s\right)$. Following the extinction test, all rats were immediately euthanized and brain tissue was collected and prepared for bFGF immunohistochemical analysis. 
To determine whether bFGF expression was changed following extinction of cocaine CPP in mPFC, we assessed differences in the density of cells expressing bFGF between groups in the prelimbic mPFC (PL-mPFC) and IL-mPFC (both: Sal-Ext, $n=4$; Coc-NoExt, $n=6$; Coc-Ext, $n=6$ ). Figure $2 \mathrm{~A}$ shows representative tissue collection areas (bregma, $+3.00 \mathrm{~mm}$; Paxinos and Watson 2007 ) and Figure $2 \mathrm{~B}$ shows representative images from each group. In PL-mPFC (Fig. 2C, left), a one-way ANOVA revealed no significant differences in bFGF expression between Sal-Ext, Coc-Ext, and Coc-NoExt rats $\left(F_{(2,13)}=2.92, P=n s\right)$. In IL-mPFC (Fig. 2C, right), a one-way ANOVA revealed a significant difference in bFGF expression between groups $\left(F_{(2,13)}=4.28, P=0.037\right)$. Post hoc tests confirmed that Coc-NoExt rats had significantly higher bFGF expression than Sal-Ext rats $(P=0.040)$ and Coc-Ext rats $(P=0.020)$, but Sal-Ext rats were not different from Coc-Ext rats $(P=n s)$. Thus, bFGF expression was increased following cocaine CPP conditioning in IL-mPFC, but not PL-mPFC, and this increase was reversed by extinction.

Next, to determine whether bFGF expression in NAc-Core or -Shell was changed following extinction, we analyzed expression between groups (both: Sal-Ext, $n=4$; Coc-NoExt, $n=6$; Coc-Ext, $n=6$ ). Figure $2 \mathrm{D}$ shows a representative area of tissue collection for NAc-Core and -Shell (bregma, +2.52 mm; Paxinos and Watson 2007), and Figure 2E shows representative images from each group. In NAc-Core (Fig. 2F, left), a one-way ANOVA revealed a significant difference in bFGF expression between groups $\left(F_{(2,13)}=5.77, P=0.016\right)$. Post hoc tests confirmed that Coc-NoExt rats had significantly higher bFGF expression than Sal-Ext rats $(P=0.009)$ and Coc-Ext rats $(P=0.020)$, but Sal-Ext rats were not different from Coc-Ext rats $(P=n s)$. In NAc-Shell (Fig. 2F, right), a one-way ANOVA revealed a significant difference in bFGF expression between groups $\left(F_{(2,13)}=4.32, P=0.036\right)$. Post hoc tests confirmed that Coc-NoExt rats had significantly higher bFGF expression than Sal-Ext rats $(P=0.022)$ and Coc-Ext rats $(P=0.034)$, but Sal-Ext rats were not different from Coc-Ext rats $(P=\mathrm{ns})$. Thus, bFGF expression was increased following cocaine $\mathrm{CPP}$ conditioning in NAc-Core and -Shell, and this increase was reversed by extinction.

\section{bFGF expression is increased in BLA following extinction, but is unchanged in Hipp}

To determine whether bFGF expression was altered in BLA following extinction, we analyzed expression between groups (Sal-Ext, $n=4$; Coc-NoExt, $n=6$; Coc-Ext, $n=6$ ). Figure $3 \mathrm{~A}$ shows a representative area of tissue collection for BLA (bregma, $-2.28 \mathrm{~mm}$; Paxinos and Watson 2007), and Figure 3B shows representative images for each group. In BLA, a one-way ANOVA revealed a significant
A

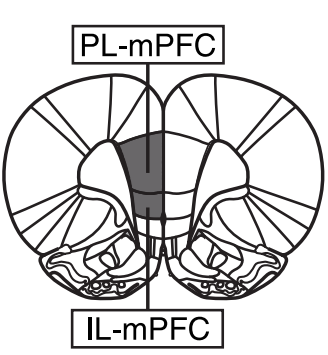

D

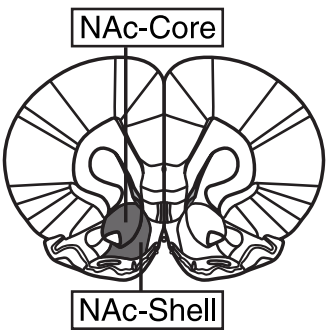

B

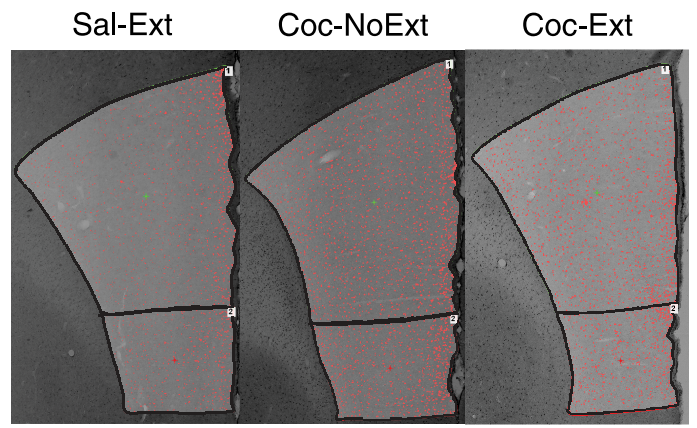

E

C

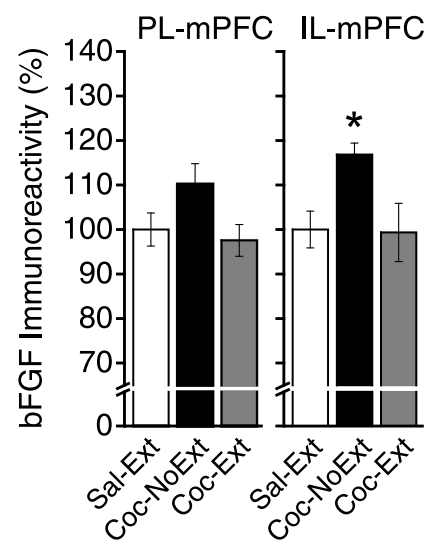

$\mathbf{F}$

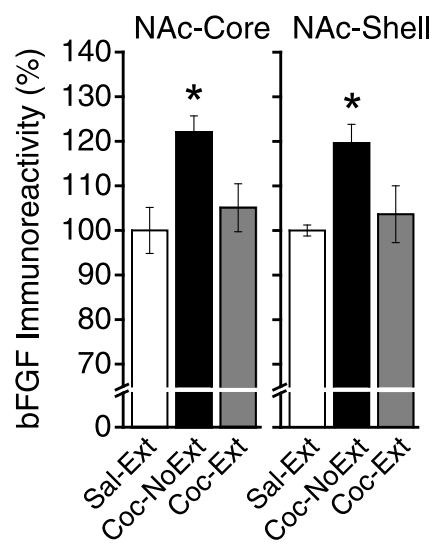

Figure 2. bFGF expression was increased in IL-mPFC and NAc-Core and -Shell following acquisition of cocaine CPP, an effect reversed by extinction. Representative areas of tissue collection containing ( $A$ ) PL-mPFC and IL-mPFC (Sal-Ext, $n=4$; Coc-NoExt, $n=6$; Coc-Ext, $n=6$ ). (B) Representative image and region of interest (ROI) analyzed from each group containing PL-mPFC and IL-mPFC. (C) bFGF expression was not significantly different between groups in PL-mPFC. However, in IL-mPFC, bFGF expression was increased in Coc-NoExt rats compared to Sal-Ext and Coc-Ext rats. $(D)$ Representative areas of tissue collection containing $(A)$ NAc-Core and-Shell (Sal-Ext, $n=4$; Coc-NoExt, $n=6$; Coc-Ext, $n=6$ ). (E) Representative image and ROI analyzed from each group containing NAc-Core and -Shell. $(F)$ bFGF expression was increased in Coc-NoExt rats compared to Sal-Ext and Coc-Ext rats, in NAc-Core and -Shell. Error bars are \pm SEM. Data normalized to \% Sal-Ext. $\left({ }^{*}\right) P<0.05$. 
A

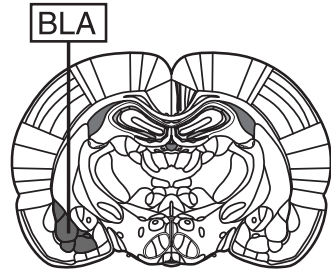

B

Sal-Ext
Coc-NoExt

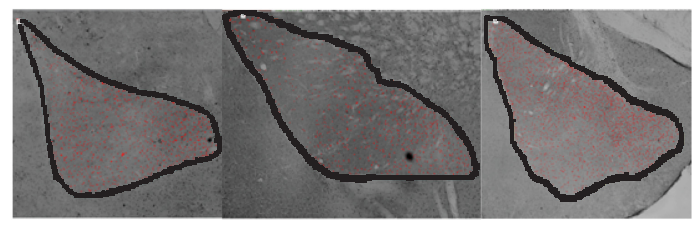

C

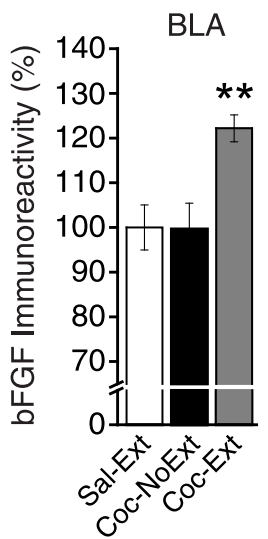

Figure 3. bFGF expression was increased in BLA following extinction. $(A)$ Representative areas of tissue collection containing BLA. ( $B$ ) Representative image and ROI analyzed from each group containing BLA (Sal-Ext, $n=4$; Coc-NoExt, $n=6$; Coc-Ext, $n=6)$. (C) bFGF expression was significantly increased in Coc-Ext rats compared to Sal-Ext and Coc-NoExt rats, in BLA. Error bars are \pm SEM. Data normalized to \% Sal-Ext. ( $\left.{ }^{\star *}\right) P<0.01$.

difference in bFGF expression between groups $\left(F_{(2,13)}=7.75, P=\right.$ 0.006; Fig. 3C). Post hoc tests confirmed that Coc-Ext rats had significantly higher bFGF expression than Coc-NoExt rats $(P=0.004)$ and Sal-Ext rats $(P=0.008)$, but Coc-NoExt rats were not different from Sal-Ext rats $(P=\mathrm{ns})$. Therefore, bFGF expression was increased in BLA following extinction of cocaine CPP, but not changed following cocaine CPP conditioning.

Finally, to determine whether extinction changed bFGF expression in dorsal Hipp (dHipp) or ventral Hipp (vHipp), we analyzed expression between groups (both: Sal-Ext, $n=4$; Coc-NoExt, $n=5$; Coc-Ext, $n=6$ ). Figure $4 \mathrm{~A}$ shows a representative area of tissue collection for dHipp (bregma, $-2.92 \mathrm{~mm}$; Paxinos and Watson 2007), and Figure $4 \mathrm{~B}$ shows representative images from each group. In dHipp, a one-way ANOVA revealed no significant differences in bFGF expression between groups $\left(F_{(2,12)}=2.39, P=n s ;\right.$ Fig. $\left.4 \mathrm{C}\right)$. Finally, for vHipp, Figure $4 \mathrm{D}$ shows a representative area of tissue collection (bregma, $-5.04 \mathrm{~mm}$; Paxinos and Watson 2007) and Figure $4 \mathrm{E}$ shows representative images from each group. In vHipp, a one-way ANOVA revealed no significant differences in bFGF expression between groups $\left(F_{(2,12)}=0.30, P=n s ;\right.$ Fig. $\left.4 F\right)$. Therefore, bFGF expression was not changed in either dHipp or vHipp following cocaine CPP conditioning or extinction.

\section{Discussion}

We found that bFGF is differentially expressed in a region-specific manner following cocaine seeking and extinction. In IL-mPFC, NAc-Core, and NAc-Shell, bFGF expression was significantly up-regulated following cocaine CPP conditioning, but expression decreased to baseline levels following extinction. Conversely, in BLA, bFGF expression was unaltered following cocaine CPP conditioning, but was increased following extinction. Finally, no significant changes in bFGF expression were observed in PL-mPFC, dHipp, or vHipp. Overall, these results indicate that bFGF expression is differentially regulated in reward- and learning-related brain regions following drug seeking or extinction, and suggests neural plasticity in circuits mediating different aspects of drug-cue associative learning and extinction.

Consistent with previous findings (Flores et al. 1998; Flores and Stewart 2000; Fumagalli et al. 2006; Hafenbreidel et al. 2015; Flagel et al. 2016), cocaine use and drug-associated learning specifically affected bFGF in IL-mPFC and NAc. These regions have reciprocal projections (Sesack et al. 1989; Groenewegen et al. 1999) and differentially mediate drug seeking. Specifically, projections between PL-mPFC and NAc-Core are thought to drive drug seeking, whereas the projections between IL-mPFC and NAc-Shell are thought to inhibit drug seeking following extinction (McFarland et al. 2003, 2004; LaLumiere and Kalivas 2008; Peters et al. 2009). Here we replicated our previous findings in IL-mPFC (Hafenbreidel et al. 2015), but did not see a significant change in PL-mPFC. It is possible that cocaine CPP increased bFGF expression transiently in PL-mPFC following conditioning, but expression was down-regulated by the time point selected to examine the effect of extinction. Others (Fumagalli et al. 2006) have reported increased bFGF expression in mPFC up to $72 \mathrm{~h}$ following five daily cocaine injections, but they did not differentiate between PL-mPFC and IL-mPFC. Similarly, we saw increased bFGF expression in NAc-Core and -Shell following drug seeking (Coc-NoExt), and a reversal of this increase following extinction. These results suggest, despite the putatively opposing roles of NAc subregions, that bFGF expression is regulated similarly in the NAc-Core and -Shell during drug seeking and extinction.

Interestingly, we found that bFGF expression was increased in BLA following extinction. The BLA is necessary for reconsolidation, or restabilization, of drug-associated memories following retrieval (Otis et al. 2013), drug-associated memory storage (Young et al. 2016), and stress- or cue-induced reinstatement (Erb et al. 2001; Kantak et al. 2002; Leri et al. 2002; McLaughlin and See 2003; McFarland et al. 2004; Hiranita et al. 2006), but not cocaine-induced reinstatement (McFarland and Kalivas 2001) or extinction in a self-administration paradigm (Peters et al. 2008). Thus, our results demonstrating the increased bFGF expression following extinction, but not following drug seeking, in BLA were surprising. However, BLA does have a role in extinction of conditioned fear (Quirk and Mueller 2008; Maren 2015), and infusions of bFGF into BLA can facilitate extinction (Graham and Richardson 2011a,b). These results, in combination with ours, suggest that bFGF has a unique role in BLA during extinction of behaviors driven by associative memories.

Our lack of significant findings in dHipp was also surprising, considering the important role of dHipp in contextual learning (Fanselow and Dong 2010) and reported increases in dHipp bFGF expression following psychostimulant administration (Fumagalli et al. 2006). These increases are transient, however, as bFGF is increased for only $2 \mathrm{~h}$ following five daily cocaine injections, and expression is not significantly different by $72 \mathrm{~h}$ after the final injection (Fumagalli et al. 2006). Our rats were euthanized $\sim 72-96 \mathrm{~h}$ 
A

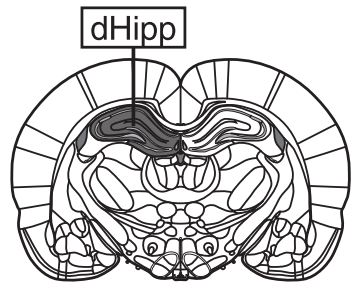

D

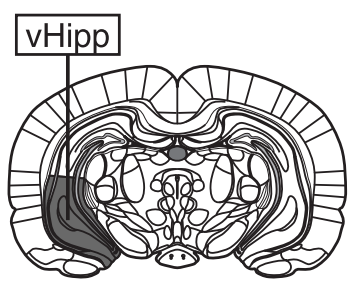

B

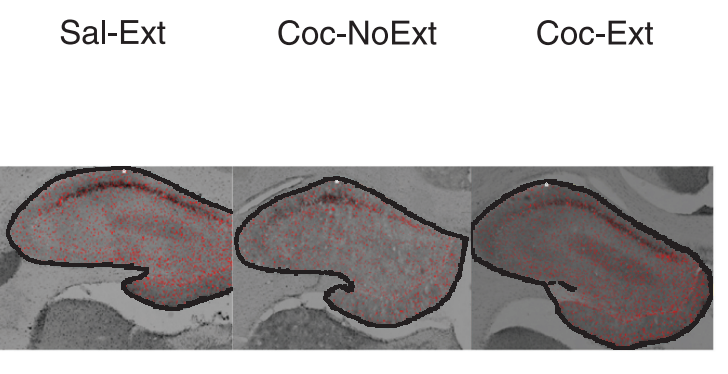

E

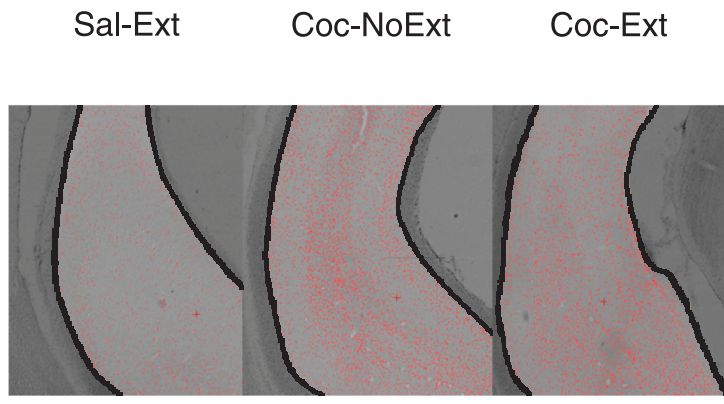

C

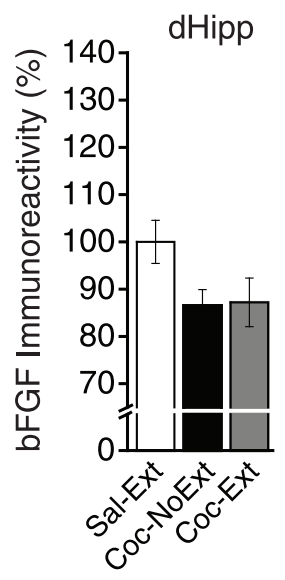

$\mathbf{F}$

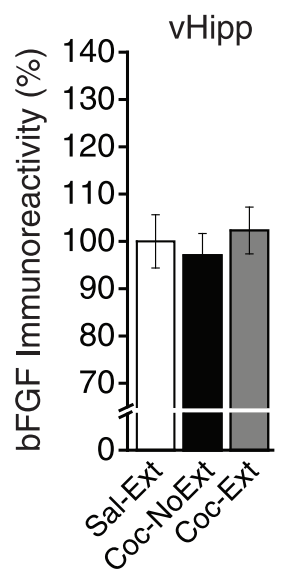

Figure 4. bFGF expression was unchanged in dHipp and vHipp. (A) Representative areas of tissue collection containing dHipp (Sal-Ext, $n=4 ;$ Coc-NoExt, $n=5$; Coc-Ext, $n=6$ ). (B) Representative image and ROI analyzed from each group containing dHipp. (C) bFGF expression was not significantly different between groups in dHipp. (D) Representative areas of tissue collection containing vHipp (Sal-Ext, $n=4$; Coc-NoExt, $n=5 ;$ Coc-Ext, $n=6$ ). (E) Representative image and ROI analyzed from each group containing vHipp. $(F)$ bFGF expression was not significantly different between groups in vHipp. Error bars are \pm SEM. Data normalized to \% Sal-Ext.

after the final cocaine injection (temporal variability arose due to the counterbalanced sequence of cocaine or saline administration on the final conditioning day, in addition to $2 \mathrm{~d}$ of extinction and $1 \mathrm{~d}$ for the extinction test). Therefore, we likely missed the transient increase in expression following cocaine injections during conditioning. Furthermore, our data suggest that neither cocaine-associated learning nor extinction in the CPP paradigm result in significant prolonged changes to bFGF expression in dHipp or vHipp.

Growth factors, such as bFGF, are associated with stimulantinduced structural and functional neural plasticity in reward- and learning-related brain regions. These plastic changes may sustain long-lasting drug-associated memories and therefore promote persistent drug-seeking behavior. For example, bFGF can enhance neurite outgrowth following stimulant administration (Mueller et al. 2006), increase expression of GluR1-containing AMPA receptors (Cheng et al. 1995; Chew et al. 1997), and promote long-term potentiation (Ishiyama et al. 1991; Zhao et al. 2007). bFGF can conversely also decrease NMDA receptor expression (Mattson et al. 1993), inhibit voltage-gated $\mathrm{Na}^{+}$(Hilborn et al. 1998) and $\mathrm{K}^{+}$currents, and decrease evoked action potentials (Cuppini et al. 2009). Together, these findings indicate that the effects of bFGF can be varied and the collective influence of bFGF on neural plasticity can be nuanced. Functionally, systemic bFGF administration can facilitate later acquisition of cocaine selfadministration (Turner et al. 2009), and bFGF in VTA is necessary for amphetamine-induced increases in locomotion and sensitization (Flores et al. 2000). Moreover, neutralizing bFGF in IL-mPFC can facilitate extinction following cocaine self-administration (Hafenbreidel et al. 2015), and neutralizing bFGF in the dorsal striatum can reduce alcohol seeking and reinstatement (Even-Chen et al. 2017). However, intra-BLA bFGF infusions can facilitate extinction of conditioned fear (Graham and Richardson 2011a,b). Whether bFGF induces neural plasticity that promotes (e.g., facilitated extinction of conditioned fear) or disrupts new learning (e.g., perseverative drug seeking) appears to be region specific (e.g., BLA versus IL-mPFC).

As suggested, certain reward- and learning-related brain regions may be specifically vulnerable to changes in bFGF elevations (Fig. 5), which could drive long-term plasticity and persistent drug seeking. In agreement with our previous findings (Hafenbreidel et al. 2015), we found increased bFGF expression in IL-mPFC and NAc-Core and -Shell following cocaine CPP conditioning (Fig. 5, left, black), which suggests that bFGF-associated adaptations within these brain regions promotes the expression of cocaine CPP. Similarly, we also found that bFGF expression was decreased in these brain regions following extinction (Fig. 5, right, white), 

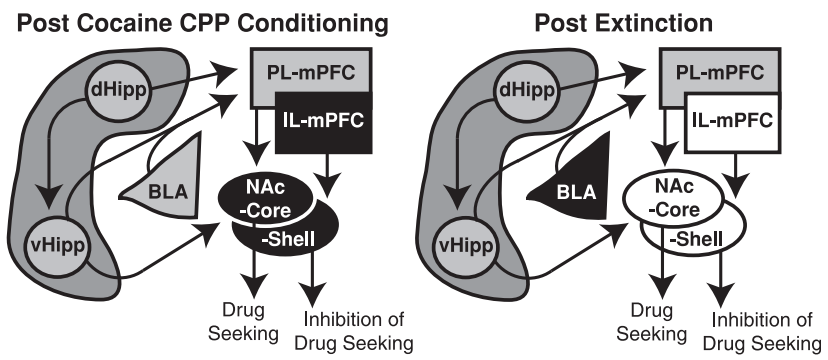

Figure 5. bFGF expression is differentially altered following cocaine seeking or extinction in drug- and learning-related brain regions. A schematic depiction of interacting brain regions that are associated with drug-associated learning and extinction, and how bFGF expression is altered in them following cocaine seeking (left), extinction (right), or both. bFGF expression in IL-mPFC, NAc-Core, and NAc-Shell is increased following drug seeking (Coc-NoExt; left; black), which is reversed following extinction (Coc-Ext; right; white). Conversely, bFGF expression is increased following extinction in BLA (Coc-Ext; right; black), but is unchanged following drug seeking (Coc-NoExt; left; gray). Finally, bFGF expression is not significantly changed in PL-mPFC, dHipp, or vHipp (left and right; gray). Gray=no change in expression; white=decrease in expression; black $=$ increase in expression.

which suggests that the decrease in expression permits adaptations that inhibit the expression of a cocaine CPP. However, increased bFGF expression may elsewhere be adaptive, as we found increased bFGF expression in BLA following extinction of cocaine CPP (Fig. 5 , right, black), an effect that agrees with previous research demonstrating that bFGF can facilitate extinction of conditioned fear (Graham and Richardson 2011a,b). Finally, bFGF may not have a prominent role in all brain regions following cocaine-associated learning, as significant changes in bFGF expression were not observed in either subregion of the hippocampus or PL-mPFC (Fig. 5 , gray). Overall, our data suggest differential bFGF regulation in a region-specific manner that can modulate either drug seeking or extinction. Future studies should examine bFGF expression immediately following cocaine CPP conditioning to determine a timeline of expression changes. Moreover, the specific cell types and output pathways regulated by bFGF, underlying mechanisms, and their functional roles should be characterized.

In conclusion, we found that bFGF expression is altered in a region-specific manner following cocaine seeking and extinction. Previous research had not examined simultaneous bFGF expression changes across relevant brain regions following cocaine CPP conditioning with or without subsequent extinction. Our results give insights into brain-wide bFGF dynamics that might mediate drug-cue associative learning and extinction. Specifically, our results suggest a contrasting role of increased bFGF expression in IL-mPFC and NAc versus BLA during cocaine seeking and extinction, and future studies should investigate the cellular and behavioral consequences of these differential expression patterns. Overall, these results suggest a modulatory role of bFGF in substance use disorder, and understanding how bFGF uniquely mediates drug seeking and extinction in specific brain regions could result in the development of novel therapeutics to enhance extinction-based therapies and help reduce relapse rates.

\section{Materials and Methods}

\section{Subjects}

Male Long-Evans rats (Harlan) weighing 175-200 g at study onset were housed and handled as previously described (Otis and Mueller 2011). Protocols were approved by the Institutional Animal Care and Use Committee at the University of Wisconsin-
Milwaukee, in compliance with National Institute of Health guidelines.

\section{Drugs}

Cocaine $\mathrm{HCl}$ (NIDA) was dissolved in sterile $0.9 \%$ saline at a concentration of $10 \mathrm{mg} / \mathrm{mL}$, and administered at a dose of $10 \mathrm{mg} / \mathrm{kg}$ (i.p.).

\section{Place preference apparatus}

Behavioral testing and conditioning were conducted as previously described (Mueller and Stewart 2000; Otis and Mueller 2011). Briefly, a three-chamber apparatus was used, in which two contextually distinct larger conditioning chambers were separated by a smaller center chamber. Rats had access to all three chambers during pre- and post-conditioning sessions, but were isolated to one of the larger chambers during conditioning. Time was recorded during CPP trials by four infrared photobeams located in the larger chambers, and total photobeam breaks made in all three chambers were used to record locomotor activity. During all phases of the experiments, the room and chambers were kept in semidarkness.

\section{Behavioral testing}

Baseline preferences were determined during a pretest as previously described (Otis and Mueller 2011). Briefly, during a pretest, rats were placed into the center chamber with free access to all three chambers for 15-min, and time spent in each chamber was recorded. A one-way ANOVA revealed a significant effect of chamber $\left(F_{(2,45)}=99.046, \quad P<0.0001\right)$, and post hoc analysis confirmed that an equivalent amount of time was spent in the conditioning chambers $(P>0.05)$, but less time was spent within the center chamber than in either conditioning chamber $(P s<0.0001)$. As rats spent equivalent time in the conditioning chambers, but less in the center chamber, an unbiased procedure was used. Additionally, all three groups displayed similar locomotor behavior during the pretest before conditioning, as a one-way ANOVA did not reveal a significant difference between groups in total photobeam breaks made in all three chambers $\left(F_{(2,13)}=2.51, P=0.120, P=\mathrm{ns}\right)$. Rats were matched into two groups, in which rats were conditioned to associate one chamber, but not the other, with cocaine in a counterbalanced manner over $8 \mathrm{~d}$. An additional control group was included, which was conditioned to associate both chambers with saline. Cocaine or saline injections were given immediately prior to each 20-min conditioning session, during which the rats were confined to their respective chambers.

To determine the effect of extinction of drug seeking on bFGF protein expression, following conditioning, saline-treated controls and half of the cocaine-treated group were given two daily CPP sessions (Sal-Ext, Coc-Ext, respectively) during which they were placed in the center chamber and given free access to the entire apparatus for 30-min to induce extinction. The other half of the experimental group was home-cage confined (Coc-NoExt). A cocaine CPP was defined when significantly more time was spent in the previously cocaine-paired chamber. Following the last extinction day, all rats were tested for extinction during a 30-min session. Immediately following the extinction test, rats were overdosed with sodium pentobarbital ( $120 \mathrm{mg} / \mathrm{kg}$, i.p.) and perfused transcardially with $150 \mathrm{~mL}$ of phosphate-buffered saline (PBS) followed by $150 \mathrm{~mL}$ of $4 \%$ paraformaldehyde in $0.1 \mathrm{M} \mathrm{PB}$ solution. Brains were extracted and stored at $4{ }^{\circ} \mathrm{C}$ in $4 \%$ paraformaldehyde overnight, then submerged in $30 \%$ sucrose for subsequent immunohistochemistry (IHC) processing and analysis.

\section{Immunohistochemistry and imaging}

Coronal sections $(50 \mu \mathrm{m})$ from each group that contained mPFC, NAc, BLA, dHipp, and vHipp were collected and processed with IHC to measure bFGF cellular expression, as previously described (Mueller et al. 2006). Briefly, sections were quenched in 30\% hydrogen peroxide and phosphate buffer $(\mathrm{PB})$, preblocked in normal goat serum and triton TBS for 30-min, and incubated in a 1:500 concentration of primary anti-bFGF (Millipore) overnight. Next, 
sections were incubated in anti-mouse secondary for 1-h, followed by incubation in Vectastain standard ELITE ABC kit for 2-h, before undergoing staining with $\mathrm{DAB}$ and $\mathrm{NiCl}_{2}$. Processed sections were imaged under a Nikon Eclipse 55i microscope using Nikon NIS Elements software. Next, regions of interest were defined (Paxinos and Watson 2007), and the total number of bFGF-immunoreactive cells in each region was quantified bilaterally in a semimanual manner across three sections to calculate average cell densities per region. Researchers were blind to conditions throughout the analysis. Data are expressed as percent immunoreactivity relative to Sal-Ext.

\section{Data analysis}

Drug-seeking behavior during each CPP session was analyzed by comparing time spent in the previously cocaine-paired, salinepaired, and center chambers using a one-way ANOVA. To examine within session behavior, a two-way repeated measures ANOVA was used to examine differences in time spent in each chamber during 3-min bins. For IHC analysis, average cell densities (number of bFGF expressing cells per $\mathrm{mm}^{2}$ ) were analyzed using a one-way ANOVA. Due to tissue damage, some samples were discarded. Final group sizes for each brain region are reported in their respective results section and figure captions. Locomotor activity (total photobeam breaks made in all three chambers during the pretest session) was analyzed using a one-way ANOVA. All post hoc tests were conducted, when appropriate, using Fisher's least significant difference (LSD) test.

\section{Acknowledgments}

We thank Dr. Robert C. Twining for advice with statistical analysis. This study was supported by NIH R01-DA038042 (D.M.), a grant from the Puerto Rico Science, Technology and Research Trust (D.M.), and NIH RCMI Program grant G12MD007579. The authors declare no conflict of interest.

\section{References}

Cheng B, Furukawa K, O'Keefe JA, Goodman Y, Kihiko M, Fabian T, Mattson MP. 1995. Basic fibroblast growth factor selectively increases AMPA-receptor subunit GluR1 protein level and differentially modulates $\mathrm{Ca}^{2+}$ responses to AMPA and NMDA in hippocampal neurons. J Neurochem 65: 2525-2536.

Chew LJ, Fleck MW, Wright P, Scherer SE, Mayer ML, Gallo V. 1997. Growth factor-induced transcription of GluR1 increases functional AMPA receptor density in glial progenitor cells. I Neurosci 17: 227-240.

Cuppini C, Ambrogini P, Lattanzi D, Ciuffoli S, Cuppini R. 2009. FGF2 modulates the voltage-dependent $\mathrm{K}^{+}$current and changes excitability of rat dentate gyrus granule cells. Neurosci Lett 462: 203-206.

Ehrman RN, Robbins SJ, Childress AR, O’Brien CP. 1992. Conditioned responses to cocaine-related stimuli in cocaine abuse patients. Psychopharmacology (Berl) 107: 523-529.

Erb S, Salmaso N, Rodaros D, Stewart J. 2001. A role for the CRF-containing pathway from central nucleus of the amygdala to bed nucleus of the stria terminalis in the stress-induced reinstatement of cocaine seeking in rats. Psychopharmacology (Berl) 158: 360-365.

Even-Chen O, Sadot-Sogrin Y, Shaham O, Barak S. 2017. Fibroblast growth factor 2 in the dorsomedial striatum is a novel positive regulator of alcohol consumption. J Neurosci 37: 8742-8754.

Fanselow MS, Dong HW. 2010. Are the dorsal and ventral hippocampus functionally distinct structures? Neuron 65: 7-19.

Flagel SB, Chaudhury S, Waselus M, Kelly R, Sewani S, Clinton SM, Thompson RC, Watson SJ Jr, Akil H. 2016. Genetic background and epigenetic modifications in the core of the nucleus accumbens predict addiction-like behavior in a rat model. Proc Natl Acad Sci 113: E2861-E2870.

Flores C, Stewart J. 2000. Changes in astrocytic basic fibroblast growth factor expression during and after prolonged exposure to escalating doses of amphetamine. Neuroscience 98: 287-293.

Flores C, Rodaros D, Stewart J. 1998. Long-lasting induction of astrocytic basic fibroblast growth factor by repeated injections of amphetamine: blockade by concurrent treatment with a glutamate antagonist. J Neurosci 18: 9547-9555.

Flores C, Samaha AN, Stewart J. 2000. Requirement of endogenous basic fibroblast growth factor for sensitization to amphetamine. J Neurosci 20: RC55.
Fumagalli F, Pasquale L, Racagni G, Riva MA. 2006. Dynamic regulation of fibroblast growth factor 2 (FGF-2) gene expression in the rat brain following single and repeated cocaine administration. J Neurochem 96: 996-1004.

Gómez-Pinilla F, So V, Kesslak JP. 1998. Spatial learning and physical activity contribute to the induction of fibroblast growth factor: neural substrates for increased cognition associated with exercise. Neuroscience 85: 53-61.

Graham BM, Richardson R. 2009a. Acute systemic fibroblast growth factor-2 enhances long-term extinction of fear and reduces reinstatement in rats. Neuropsychopharmacology 34: 1875-1882.

Graham BM, Richardson R. 2009b. Acute systemic fibroblast growth factor-2 enhances long-term memory in developing rats. Neurobiol Learn Mem 91: $424-430$.

Graham BM, Richardson R. 2010. Fibroblast growth factor-2 enhances extinction and reduces renewal of conditioned fear. Neuropsychopharmacology 35: 1348-1355.

Graham BM, Richardson R. 2011a. Fibroblast growth factor-2 alters the nature of extinction. Learn Mem 18: 80-84.

Graham BM, Richardson R. 2011b. Intraamygdala infusion of fibroblast growth factor 2 enhances extinction and reduces renewal and reinstatement in adult rats. J Neurosci 31: 14151-14157.

Groenewegen HJ, Galis-de Graaf Y, Smeets WJ. 1999. Integration and segregation of limbic cortico-striatal loops at the thalamic level: an experimental tracing study in rats. J Chem Neuroanat 16: 167-185.

Hafenbreidel M, Twining RC, Rafa Todd C, Mueller D. 2015. Blocking infralimbic basic fibroblast growth factor (bFGF or FGF2) facilitates extinction of drug seeking after cocaine self-administration. Neuropsychopharmacology 40: 2907-2915.

Hilborn MD, Vaillancourt RR, Rane SG. 1998. Growth factor receptor tyrosine kinases acutely regulate neuronal sodium channels through the src signaling pathway. J Neurosci 18: 590-600.

Hiranita T, Nawata Y, Sakimura K, Anggadiredja K, Yamamoto T. 2006. Suppression of methamphetamine-seeking behavior by nicotinic agonists. Proc Natl Acad Sci 103: 8523-8527.

Ishiyama J, Saito H, Abe K. 1991. Epidermal growth factor and basic fibroblast growth factor promote the generation of long-term potentiation in the dentate gyrus of anaesthetized rats. Neurosci Res 12: 403-411.

Kantak KM, Black Y, Valencia E, Green-Jordan K, Eichenbaum HB. 2002. Dissociable effects of lidocaine inactivation of the rostral and caudal basolateral amygdala on the maintenance and reinstatement of cocaine-seeking behavior in rats. J Neurosci 22: 1126-1136.

Kosten TR, Scanley BE, Tucker KA, Oliveto A, Prince C, Sinha R, Potenza MN, Skudlarski P, Wexler BE. 2006. Cue-induced brain activity changes and relapse in cocaine-dependent patients. Neuropsychopharmacology 31: 644-650.

LaLumiere RT, Kalivas PW. 2008. Glutamate release in the nucleus accumbens core is necessary for heroin seeking. J Neurosci 28: 3170-3177.

Leri F, Flores J, Rodaros D, Stewart J. 2002. Blockade of stress-induced but not cocaine-induced reinstatement by infusion of noradrenergic antagonists into the bed nucleus of the stria terminalis or the central nucleus of the amygdala. J Neurosci 22: 5713-5718.

Maggio R, Riva M, Vaglini F, Fornai F, Molteni R, Armogida M, Racagni G, Corsini GU. 1998. Nicotine prevents experimental parkinsonism in rodents and induces striatal increase of neurotrophic factors. J Neurochem 71: 2439-2446.

Maren S. 2015. Out with the old and in with the new: Synaptic mechanisms of extinction in the amygdala. Brain Res 1621: 231-238.

Mattson MP, Kumar KN, Wang H, Cheng B, Michaelis EK. 1993. Basic FGF regulates the expression of a functional $71 \mathrm{kDa}$ NMDA receptor protein that mediates calcium influx and neurotoxicity in hippocampal neurons. J Neurosci 13: 4575-4588.

McFarland K, Kalivas PW. 2001. The circuitry mediating cocaine-induced reinstatement of drug-seeking behavior. J Neurosci 21: 8655-8663.

McFarland K, Lapish CC, Kalivas PW. 2003. Prefrontal glutamate release into the core of the nucleus accumbens mediates cocaine-induced reinstatement of drug-seeking behavior. J Neurosci 23: 3531-3537.

McFarland K, Davidge SB, Lapish CC, Kalivas PW. 2004. Limbic and motor circuitry underlying footshock-induced reinstatement of cocaine-seeking behavior. J Neurosci 24: 1551-1560.

McLaughlin J, See RE. 2003. Selective inactivation of the dorsomedial prefrontal cortex and the basolateral amygdala attenuates conditioned-cued reinstatement of extinguished cocaine-seeking behavior in rats. Psychopharmacology (Berl) 168: 57-65.

McLellan AT, Lewis DC, O'Brien CP, Kleber HD. 2000. Drug dependence, a chronic medical illness: implications for treatment, insurance, and outcomes evaluation. JAMA 284: 1689-1695.

Mueller D, Stewart J. 2000. Cocaine-induced conditioned place preference: reinstatement by priming injections of cocaine after extinction. Behav Brain Res 115: 39-47. 
Mueller D, Chapman CA, Stewart J. 2006. Amphetamine induces dendritic growth in ventral tegmental area dopaminergic neurons in vivo via basic fibroblast growth factor. Neuroscience 137: 727-735.

Otis JM, Mueller D. 2011. Inhibition of $\beta$-adrenergic receptors induces a persistent deficit in retrieval of a cocaine-associated memory providing protection against reinstatement. Neuropsychopharmacology 36: 1912-1920.

Otis JM, Dashew KB, Mueller D. 2013. Neurobiological dissociation of retrieval and reconsolidation of cocaine-associated memory. J Neurosci 33: $1271-1281 \mathrm{a}$.

Paxinos G, Watson C. 2007. The rat brain in stereotaxic coordinates. Academic, Burlington, MA.

Peters J, LaLumiere RT, Kalivas PW. 2008. Infralimbic prefrontal cortex is responsible for inhibiting cocaine seeking in extinguished rats. J Neurosci 28: 6046-6053.

Peters J, Kalivas PW, Quirk GJ. 2009. Extinction circuits for fear and addiction overlap in prefrontal cortex. Learn Mem 16: 279-288.

Quirk GJ, Mueller D. 2008. Neural mechanisms of extinction learning and retrieval. Neuropsychopharmacology 33: 56-72.

Robinson TE, Kolb B. 1997. Persistent structural modifications in nucleus accumbens and prefrontal cortex neurons produced by previous experience with amphetamine. J Neurosci 17: 8491-8497.

Robinson TE, Kolb B. 1999. Alterations in the morphology of dendrites and dendritic spines in the nucleus accumbens and prefrontal cortex following repeated treatment with amphetamine or cocaine. Eur J Neurosci 11: 1598-1604.

Roceri M, Molteni R, Fumagalli F, Racagni G, Gennarelli M, Corsini G, Maggio R, Riva M. 2001. Stimulatory role of dopamine on fibroblast growth factor-2 expression in rat striatum. J Neurochem 76: 990-997.

Sesack SR, Deutch AY, Roth RH, Bunney BS. 1989. Topographical organization of the efferent projections of the medial prefrontal cortex in the rat: an anterograde tract-tracing study with Phaseolus vulgaris leucoagglutinin. J Comp Neurol 290: 213-242.

Srivastava N, Seth K, Srivastava N, Khanna VK, Agrawal AK. 2008. Functional restoration using basic fibroblast growth factor (bFGF) infusion in Kainic acid induced cognitive dysfunction in rat: neurobehavioural and neurochemical studies. Neurochem Res 33: 1169-1177.

Turner CA, Capriles N, Flagel SB, Perez JA, Clinton SM, Watson SJ, Akil H. 2009. Neonatal FGF2 alters cocaine self-administration in the adult rat. Pharmacol Biochem Behav 92: 100-104.

Young EJ, Blouin AM, Briggs SB, Sillivan SE, Lin L, Cameron MD, Rumbaugh G, Miller CA. 2016. Nonmuscle myosin IIB as a therapeutic target for the prevention of relapse to methamphetamine use. Mol Psychiatry 21: 615-623.

Zhao M, Li D, Shimazu K, Zhou YX, Lu B, Deng CX. 2007. Fibroblast growth factor receptor-1 is required for long-term potentiation, memory consolidation, and neurogenesis. Biol Psychiatry 62: $381-390$.

Received March 9, 2018; accepted in revised form June 11, 2018. 


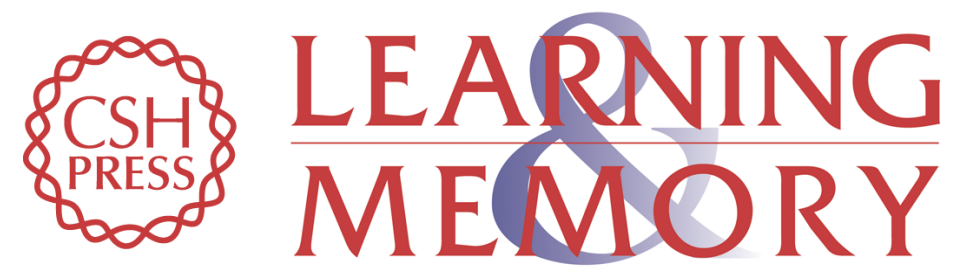

\section{bFGF expression is differentially regulated by cocaine seeking versus extinction in learning-related brain regions}

Elizabeth M. Doncheck, Madalyn Hafenbreidel, Sarah A. Ruder, et al.

Learn. Mem. 2018, 25:

Access the most recent version at doi:10.1101/Im.047530.118

References This article cites 50 articles, 20 of which can be accessed free at: http://learnmem.cshlp.org/content/25/8/361.full.html\#ref-list-1

Creative This article, published in Learning \& Memory, is available under a Creative Commons Commons License (Attribution-NonCommercial 4.0 International), as described at License http://creativecommons.org/licenses/by-nc/4.0/.

Email Alerting Receive free email alerts when new articles cite this article - sign up in the box at the Service top right corner of the article or click here. 